\title{
High-throughput Quant-iT PicoGreen assay using an automated liquid handling system
}

\author{
Kay Anantanawat ${ }^{1,2}$, Nicola Pitsch', Caroline Fromont ${ }^{1,3}$ \& Caroline Janitz*,1
}

\section{ABSTRACT}

Workflows in NGS facilities require high-standard practices and highthroughput pipelines to process the large number of samples received in a timely manner. Downstream protocols such as NGS library preparation require accurate estimation of nucleic acid concentrations, which can be achieved using fluorescent dye-based nucleic acid measurement. Here, we report a protocol for preparing a 384-well Quant-iT PicoGreen assay. The protocol allows the concentrations of 184 DNA samples to be measured simultaneously in duplicate in only $1 \mathrm{~h}$ using an Eppendorf epMotion 5075 liquid handling system. The advantages of this high-throughput approach include a reduction in both reagents (10x less reagents compared to a standard protocol) and time ( $3 \mathrm{~h}$ for 384 samples compared with 3 days).

\section{METHOD SUMMARY}

We describe a protocol for preparing a 384-well Quant-iT PicoGreen assay for utilization in a NGS pipeline. The protocol allows the concentrations of 184 DNA samples to be measured simultaneously in duplicate in only 1 h using an Eppendorf epMotion 5075 liquid handling system.

\section{KEYWORDS:}

DNA library $\cdot$ high-throughput $\cdot$ liquid handling systems $\cdot$ next-generation sequencing $\cdot$ PicoGreen assay

'The Next Generation Sequencing Facility, Western Sydney University, Sydney, Australia; ${ }^{2}$ Present address: The ithree Institute, University of Technology Sydney, Sydney, Australia; ${ }^{3}$ Present address: Department of Entomology, Cornell University, Ithaca, $N Y$, USA; *Author for correspondence: c.janitz@ westernsydney.edu.au

BioTechniques 66: 290-294 (June 2019) 10.2144/btn-2018-0172
The success of the NGS service facility relies on the quality of data generated in a timely fashion. This outcome is challenged by the quality of the starting DNA material, the complexity of library preparation and the number of samples being processed. To create high-quality NGS data, the quality of the starting nucleic acid material needs to be assessed accurately. A commonly used technique for measuring DNA is the absorbance measurement at $260 \mathrm{~nm}$ [1,2]. Although this technique is quick and easy, it is not suitable for the NGS pipeline as it is not sufficiently accurate [3,4]. A260 measurement does not differentiate between double-stranded (ds)DNA, singlestranded (ss)DNA and RNA well, and thus it gives an overestimation of the real concentration of dsDNA.

A novel approach based on fluorescence assays is more appropriate and accurate for DNA input quantification $[5,6]$ for any applications in molecular biology. This alternative method has become the gold standard for the determination of dsDNA content in DNA samples. The use of fluorescent dyes such as Quant-iT PicoGreen [6] is much more sensitive and preferred in the NGS pipeline [7]. As suggested by Dragan and colleagues, DNA interaction with PicoGreen is facilitated by structure of this dye containing the three structural elements responsible for the different types of interaction with DNA: the phenyl-quinolinium and the benzo-thiazol aromatic systems, and the dimethylaminopropyl elongated chains. It is assumed that the quinolinium group of PicoGreen intercalates into DNA. It is assumed that tight contact of the quinolinium group between basepairs is stabilized by van der Waals interactions with the DNA bases and rigidify this structural element on the DNA [8]. A PicoGreenbased DNA detection technique has many advantages over the previously used spectrophotometer (NanoDrop); they are summarized in Table 1. The fluorescence-based assay has superiority over NanoDrop in its specificity, as it can detect only dsDNA content. Moreover, it has much higher (over 1000-fold) sensitivity, allowing the detection of as little as approximately $0.5 \mathrm{ng} / \mathrm{ml}$ DNA in solution. Therefore, the fluorescence technique is affordable and provides a high-throughput option for the service facility to measure dsDNA prior to library preparation. The fluorophore used in the Quant-iT PicoGreen assay fluoresces upon being intercalated specifically with dsDNA. The fluorescence intensity of the resulting complex depends directly on the amount of target molecule in the sample and can be measured using a spectrofluorometer. The fluorescence is excited at $480 \mathrm{~nm}$, and the fluorescence emission intensity is measured at $520 \mathrm{~nm}$. Unknown sample concentrations are determined from the fluorescence readings using a regression curve generated from Lambda DNA standards measured during the same assay. Thus, the technique allows an accurate measurement of dsDNA, as it does not detect other nucleic acid molecules, such as ssDNA and RNA.

Despite the accuracy of the Quant-iT PicoGreen assay, the amount of time used to prepare the assay can be substantial. Preparation can be tedious when the number of samples to be processed is in the hundreds, which is usually the case in most service facilities. Here, we have developed a protocol to set up a PicoGreen assay in a 384-well plate using an automatic liquid handling system, epMotion 5075 (Eppendorf, Germany). The protocol allows the preparation of an assay plate for 184 samples within $40 \mathrm{~min}$, and the complete analysis is performed within $1 \mathrm{~h}$. In addition to saving time, the amount of Quant-iT reagent used in the protocol is 10-fold less than that in the original protocol, which is a great cost saving for the facility. 
Table 1. The use of fluorescence dye-based and A260 absorbance measurement for quality control in the NGS pipeline.

\begin{tabular}{|l|l|l|}
\hline Primary application & Qubit/PicoGreen & Quality \\
\hline Numbers of wavelengths used & 2 & 1 \\
\hline Signal-to-background noise & High & Low \\
\hline Sensitivity & High ( 1000x greater than Nano Drop) & Low \\
\hline Specificity for double-stranded DNA & $\begin{array}{l}\cdot \text { High } \\
\bullet \text { The dye intercalates only into double- } \\
\text { stranded DNA }\end{array}$ & $\begin{array}{l}\text { • Low } \\
\text { Single-stranded nucleic acids and nucleotides can } \\
\text { contribute to the signal } \\
\bullet \text { Inability to distinguish between DNA and RNA }\end{array}$ \\
\hline
\end{tabular}

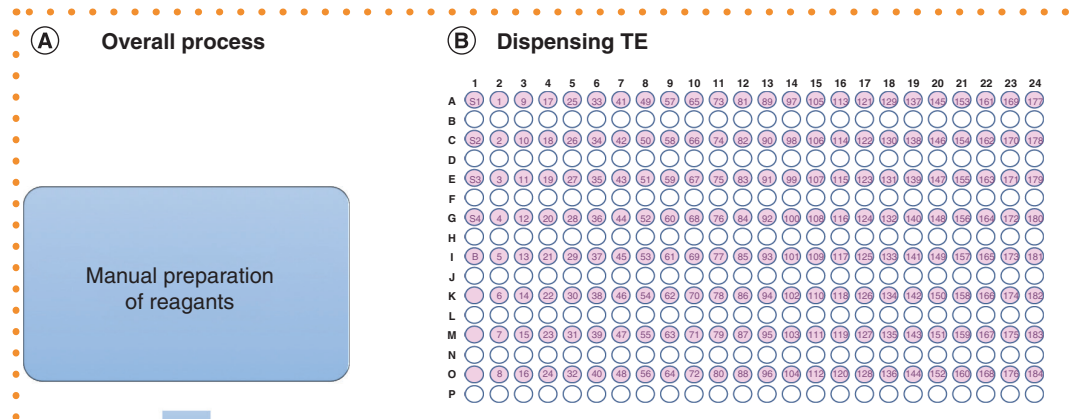

(D) Dispensing samples

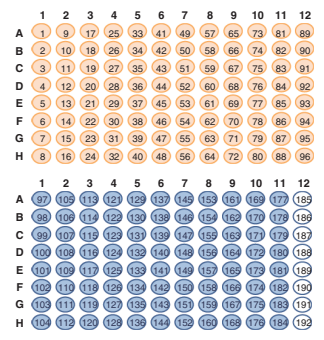

(E) Dispensing PicoGreen

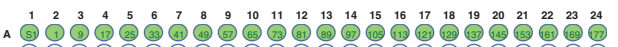

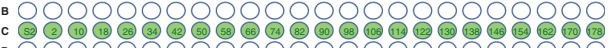

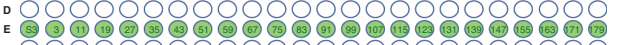

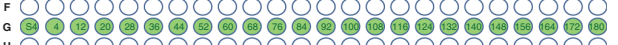

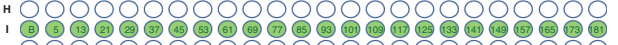
к

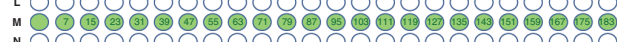

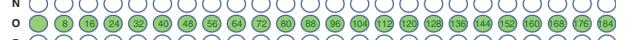

Fluorometric quantification using a plate reader
(C) Serial dilution of standards

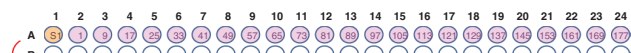

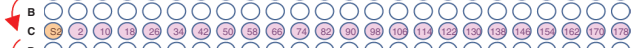

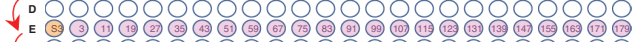

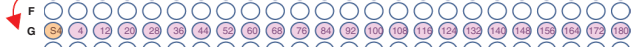

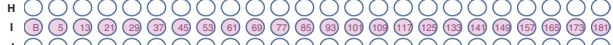

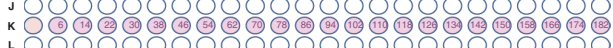

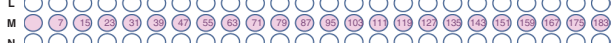

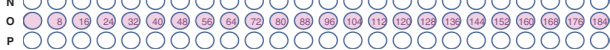

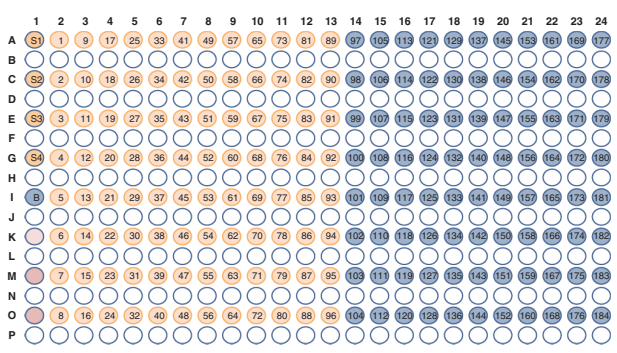

(F) Preparing duplicate

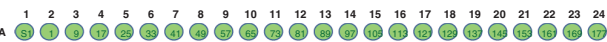

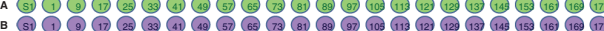

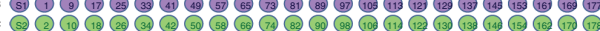

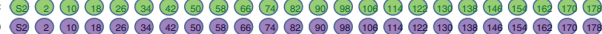

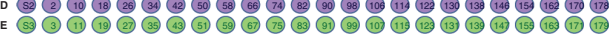

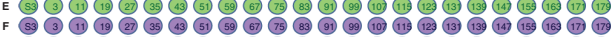

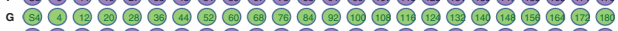

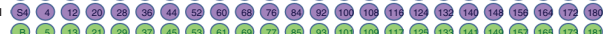

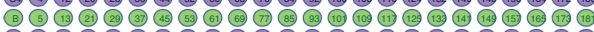

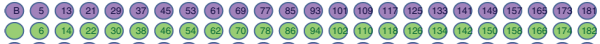

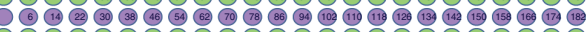

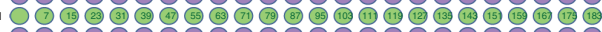

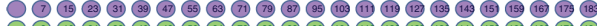

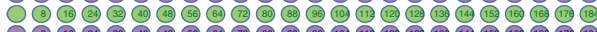

Figure 1. The diagram of the Quant-iT PicoGreen assay for the 384-well plate set up. (A) The overall set up of the assay includes manual preparation of the reagents, automated preparation of the assay plate and fluorometric quantification using a plate reader. B-F are steps of the assay plate preparation using an epMotion. Briefly, (B) $1 \times$ TE is distributed into rows of the first column for standard preparation, and $20 \mu \mathrm{l}$ of $1 \mathrm{x} T E$ is transferred into $1 \mathrm{C}$, $1 \mathrm{E}$, $1 \mathrm{G}$ and 11. A total of $19 \mu \mathrm{l}$ of $1 x$ TE is transferred into rows $A, C, E, G, I, K, M$ and $O$ of columns 2-24. (C) Standards are serially diluted in the first column of the plate. (D) $1 \mu \mathrm{l}$ of DNA samples is added into the wells containing $1 \times$ TE using a multichannel pipette. A total of 184 samples can be added to the 384 -well plate. (E) $20 \mu \mathrm{l}$ of Quant-iT PicoGreen is added to the well containing the samples. The plate is mixed at $1000 \mathrm{RPM}$ for $2 \mathrm{~min}$. (F) $20 \mu \mathrm{l}$ of the samples/ PicoGreen mix is transferred from rows $A, C, E, G, I, K, M$ and $O$ into $B, D, F, H, J, L, N$ and $P$, respectively, in the same column. The plate is then transferred to the plate reader for measurement. 


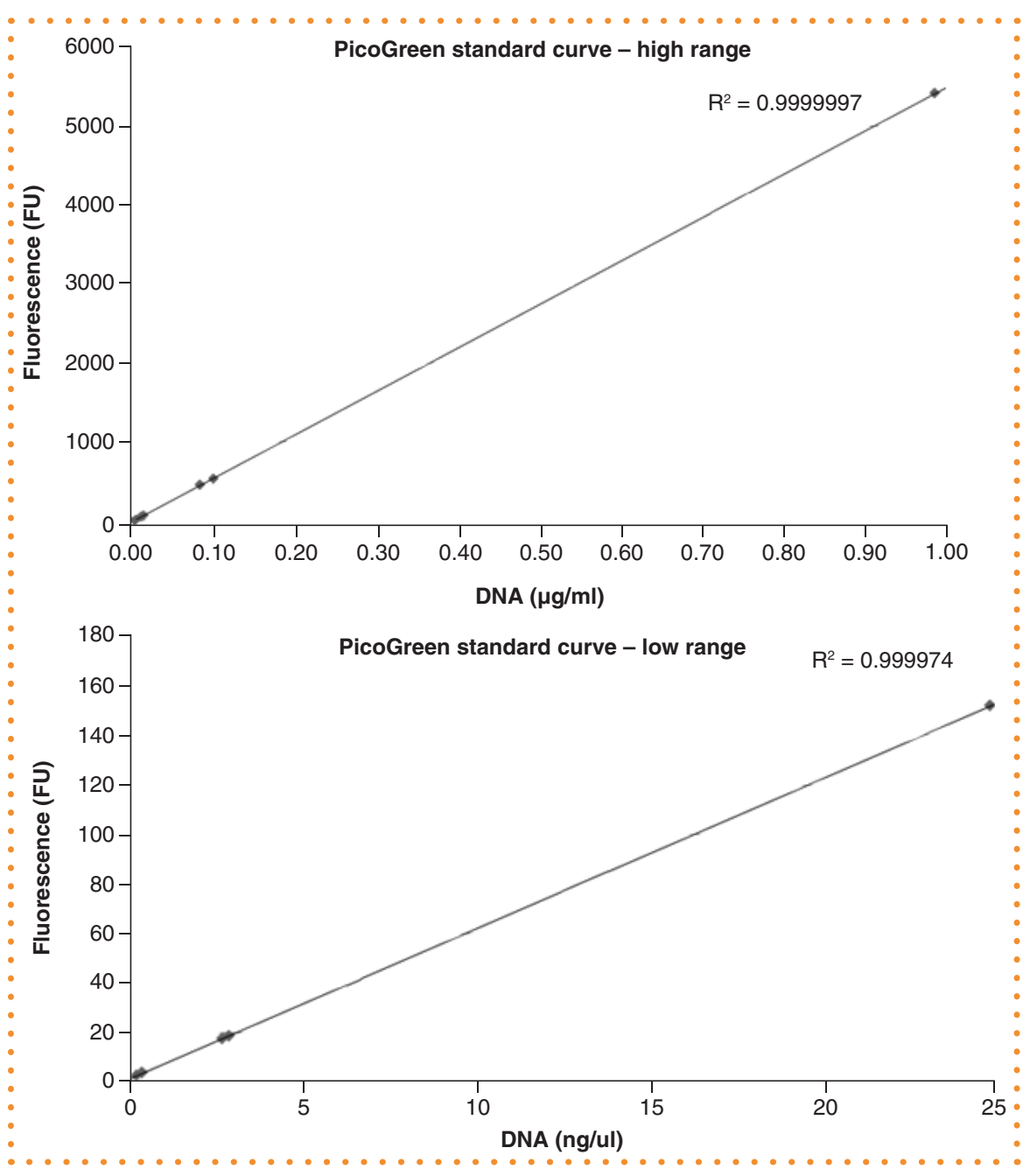

Figure 2. Detection of double-stranded DNA with the PicoGreen Reagent using the filter combination Ex 485/Em538. The assay was performed using a black 384-well plate (total volume of $20 \mu \mathrm{l} /$ well). The assay showed perfect linearity over both concentration ranges - low and high range - indicating the reliable determination of DNA concentration in both low- and high-concentration samples.

\section{PREPARING REAGENTS FOR EPMOTION}

The Quant-iT PicoGreen assay was prepared based on the manufacturer's instructions. The assay was prepared for 184 aliquots of DNA sample and five DNA standards, including one blank. The DNA sample used for aliquoting was extracted from soil using the DNeasy PowerSoil Kit (Qiagen). The DNA standard was Lambda DNA included in the Quant-iT PicoGreen dsDNA Assay Kit (ThermoFisher). Briefly, a 1x TE working solution was prepared by diluting the concentrated 20x TE buffer with nucleasefree water. The TE working solution was used as a blank and for preparing all the DNA standard dilutions and samples. For the PicoGreen working solution, the
Quant-iT PicoGreen reagent was diluted $200 x$ with TE buffer in a plastic tube. The working solution was protected using aluminum foil and was used within 1 h. 20 $\mu \mathrm{l}$ of diluted PicoGreen was needed per well. Both working solutions were transferred individually into $30 \mathrm{ml}$ reservoirs for later use in the epMotion.

Quant-iT PicoGreen assays come in two ranges: a high-range and a low-range assay. The high range generated a standard curve from $1 \mathrm{ng} / \mathrm{ml}$ to $1 \mu \mathrm{g} / \mathrm{ml}$, whereas the low range generated a standard curve from $25 \mathrm{pg} / \mathrm{ml}$ to $25 \mathrm{ng} / \mathrm{ml}$. We prepared and performed both assays separately. For the high-range assay, a small aliquot of the provided Lambda DNA standard was diluted 1:50 with TE buffer to prepare a $2 \mu \mathrm{g} / \mathrm{ml}$ DNA stock solution in TE buffer. For the low-range assay, the DNA standard was prepared by diluting the $2 \mu \mathrm{g} / \mathrm{ml}$ DNA stock solution with TE buffer in a 1:40 ratio, giving a $50 \mathrm{ng} /$ $\mu$ I DNA stock solution. The standard was set up as a $1.5-\mathrm{ml}$ tube in a $1.5-\mathrm{ml}$ tube rack for the epMotion. The serial 1:10 dilutions of the high- and low-range standards to create the calibration curve was performed using the epMotion (see below).

\section{SETTING UP THE ASSAY PLATE USING EPMOTION 5075}

\section{Dispensing TE}

Both low-range and high-range assays were prepared separately using an epMotion 5075 (Figure 1). First, 1x TE buffer was distributed into a black 384-well assay plate (Eppendorf). The distribution was performed in two steps: $20 \mu \mathrm{l}$ of $1 \mathrm{x}$ TE buffer was pipetted using a single-channel tool into positions 1C, 1E, 1G and 1 (Figure 1A). Then, $19 \mu \mathrm{l}$ of $1 \mathrm{x}$ TE was transferred into positions $A, C, E, G, I, K, M, O$ of columns 2-24 using an eight multichannel pipette (TM_50_8).

\section{Preparation of standards \& positive control}

Briefly, $22 \mu$ l of the highest concentration standard was transferred into $1 \mathrm{~A}$. Then $2.2 \mu \mathrm{l}$ of DNA was transferred into 1C (standard 2) (Figure 1B). The plate was mixed at 1000 RPM for $30 \mathrm{~s}$. Then, $2.2 \mu \mathrm{l}$ was transferred to $1 \mathrm{E}$ (standard 3 ). The mixing was repeated, and $2.2 \mu \mathrm{l}$ of standard 3 was transferred from $1 \mathrm{E}$ to $1 \mathrm{G}$. After mixing, $2.2 \mu \mathrm{l}$ of liquid from $1 G$ was discarded.

\section{Dispensing samples}

After preparing the standards, $1 \mu$ of DNA samples was transferred using the 8 multichannel tool to positions A, C, E, G, I, K, M and $\mathrm{O}$ of columns 2 onward, and each column of the 96 -well plate corresponded to a column of the 384-well plate (Figure 1C).

\section{Dispensing PicoGreen}

Then, $20 \mu$ of 1x Quant-iT mix was added into each well using an 8 multichannel tool (Figure 1D), and the plate was mixed at 1000 RPM for 2 min.

\section{Preparing duplicates}

After mixing, for each column in the 384-well 
plate, $20 \mu$ from wells $A, C, E, G, I, K, M$ and $O$ were transferred into wells $B, D, F, H, J, L$, $\mathrm{N}$ and $\mathrm{P}$ in the same column using the multichannel tool to make a replicate (Figure 1E).

\section{MEASURING THE CONCENTRATION}

The assay was measured using the BMG FLUOstar Fluorescence plate reader (BMG LABTECH, Germany) with the excitation set at $480 \mathrm{~nm}$ and the emission measured at $520 \mathrm{~nm}$. Readings were analyzed using OPTIMA Data Analysis (MARS Data Analysis Software, BMG LABTECH). The fluorescence reading of the blank was subtracted from sample raw fluorescence readings. The fluorescence readings from the standards were then used to create a calibration curve. The concentrations of the samples were then calculated based on the calibration curve. Since samples were diluted $40 x$, the measured concentrations needed to be multiplied by 40 to obtain the original sample concentrations. The average and the standard deviation for each sample were calculated from the duplicates. The same protocol was repeated using a lower range measurement of PicoGreen.

The assay plate was measured using a BMG FLUOstar, and the standard curve was generated from the fluorescence readings of the DNA standards after subtracting the blank reading (Figure 2). The results of the standards showed perfect linearity over both low- and high-concentration ranges, indicating the reliable determination of DNA concentrations in both low- and highconcentration samples.

For both low- and high-range assays, the reading of the plate was uniform (Figure 3). There was no pattern indicating that the location of the well in the plate affects the reading of the samples. The differences between duplicates were between 0.0 and $0.6 \mathrm{ng} / \mu \mathrm{l}$ for the low-range assay, although the high-range assay had wider variation between duplicates, 0.001-2.05 ng/ $\mu \mathrm{l}$ (Figure 4). The standard deviation for each sample was calculated and is shown in Figure 5.

The average concentration of DNA measured in the plate using a low range was $1.34 \mathrm{ng} / \mu \mathrm{l}$, and using a high range was $14.12 \mathrm{ng} / \mu \mathrm{l}$, with standard deviations of $0.23 \mathrm{ng} / \mu \mathrm{l}$ and $1.28 \mathrm{ng} / \mu \mathrm{l}$, respectively. $\begin{array}{llllllllllllllllllllllll}1 & 2 & 3 & 4 & 5 & 6 & 7 & 8 & 9 & 10 & 11 & 12 & 13 & 14 & 15 & 16 & 17 & 18 & 19 & 20 & 21 & 22 & 23 & 24\end{array}$

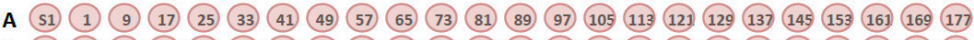

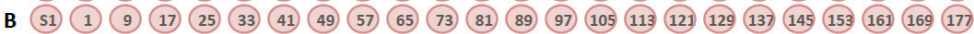

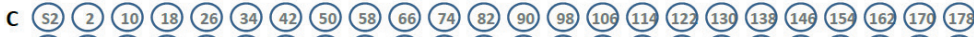

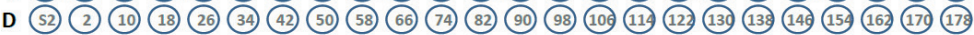

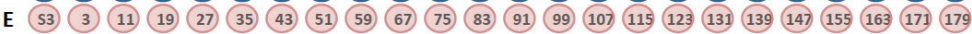

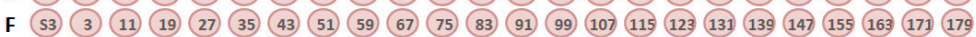

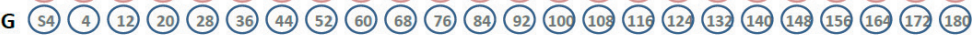

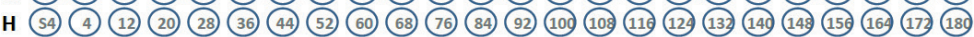

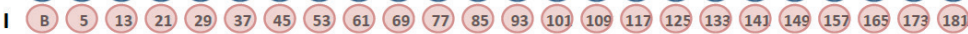

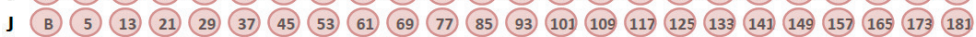
K $\bigcirc$ (6) (14) (22) (30) (38) (46) (54) (62) (70) (78) (86) (94) (102) (111) (112) (122) (132) (142) (152 (152) (16) (172) (182)

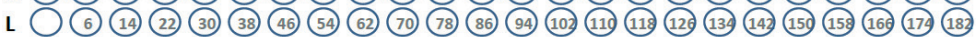

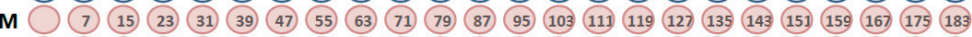

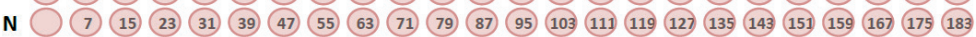

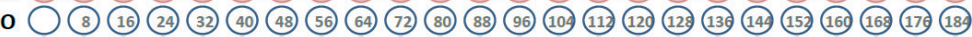
$P$ (8) (16) (24) (32) (40) (48) (56) (64) (72) (80) (82) (96) (102) (112) (122) (122) (132) (14) (152) (16) (16) (172) (18)

A. Concentration of DNA ( $\mathrm{ng} / \mathrm{\mu l}$ ) measured using high-range PicoGreen assay

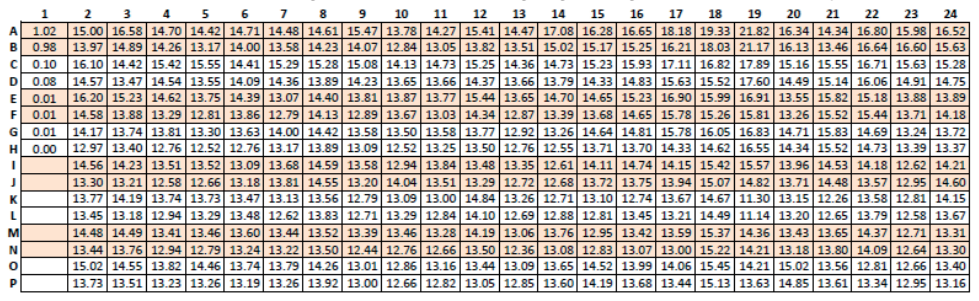

B. Concentration of DNA (ng/ $\mu \mathrm{l})$ measured using low-range PicoGreen assay

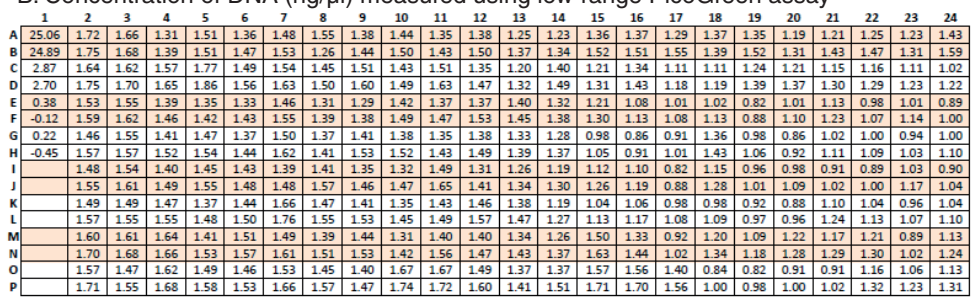

Figure 3. Concentration of DNA measured in each well in the high-range $(A)$ and the low-range (B) PicoGreen assays. The plate layout is shown above.

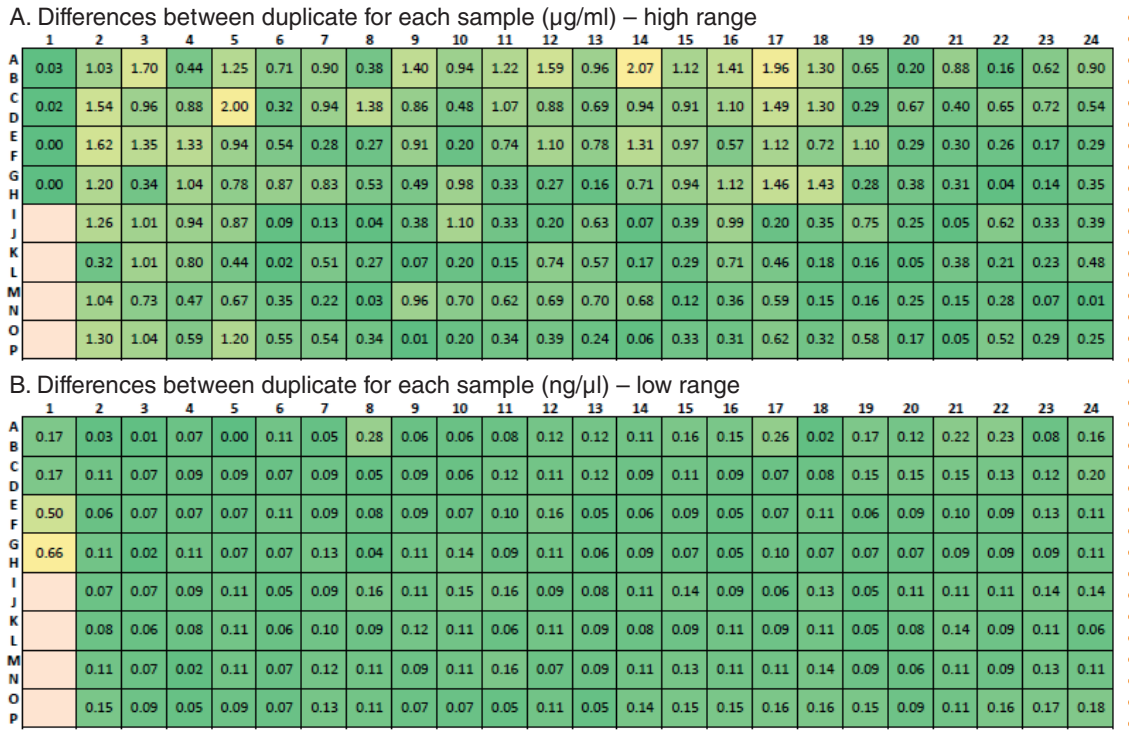

Figure 4. Differences between duplicates for each sample $(\mathrm{ng} / \mu \mathrm{l})$ are shown in color-coded boxes; green represents samples with the lowest differences between duplicates, whereas yellow represents samples with the highest differences between duplicates. (A) High range, (B) low range. 


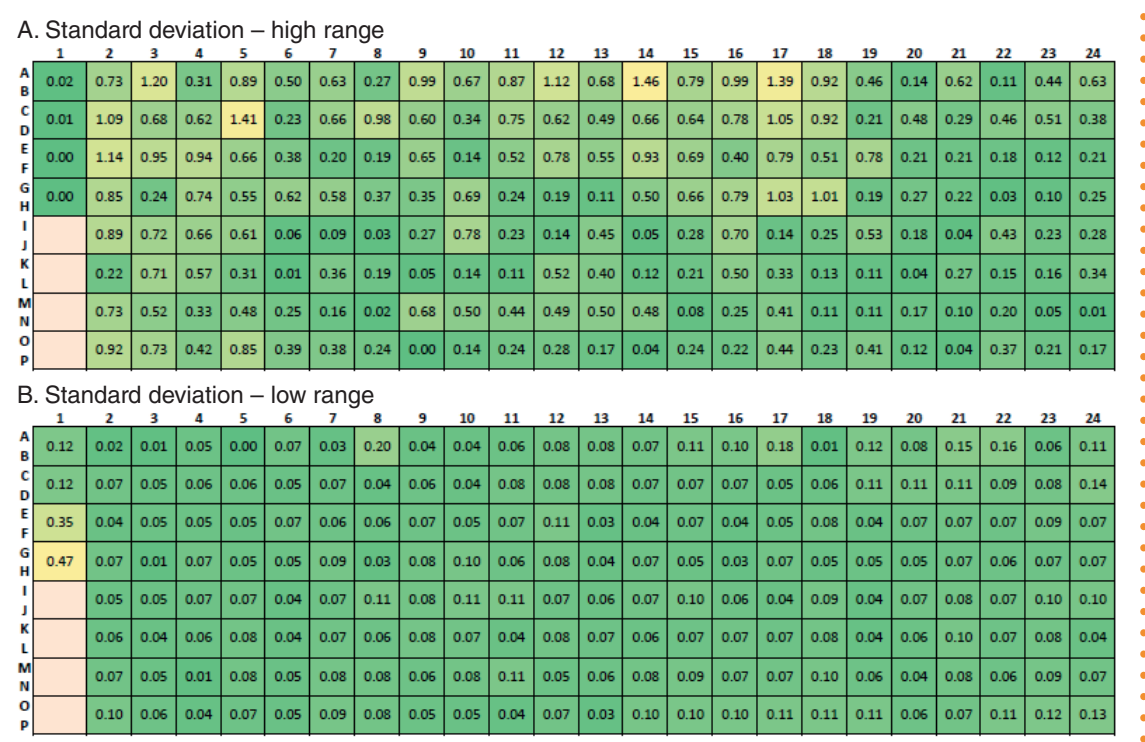

Figure 5. Standard deviation calculated from duplicates for each sample. Green represents the lowest values, whereas yellow represents the highest values. (A) High range, (B) low range.

\section{- Quant-iT PicoGreen is a technique} that is used to measure dsDNA with high accuracy and sensitivity. Together with the use of epMotion, we have successfully created a protocol that enables the accurate measurement of DNA in a highthroughput fashion. The protocol allows 184 samples to be measured in less than $1 \mathrm{~h}$. The protocol also gave highly reproducible data (data not shown).

The high-range assay had higher variation between duplicates because the effect of the pipetting error can be easily detected by the high concentration of the samples used in the assay. For example, if the concentration of the DNA is $15 \mathrm{ng} / \mu \mathrm{l}$ and $1 \mu \mathrm{l}$ of DNA is used, a pipetting error of $0.5 \%$ can increase the amount of DNA from $15 \mathrm{ng}$ to $15.75 \mathrm{ng}$ in the reaction. The difference in $0.75 \mathrm{ng}$ between duplicates can be captured by the fluorescence readings, as the PicoGreen assay is very sensitive. This effect was not captured in the low-range assay, as the concentration of the samples used was low $(1.5 \mathrm{ng} / \mu \mathrm{l})$ and the pipetting error of $0.5 \%$ has a minor effect on the DNA amount to the point that it is not detected by the assay. In conclusion, even though it appears that the high-range assay showed higher variation between duplicates, it is of no significance.

Compared with the manufacturer's protocol, which requires $200 \mu$ l of reagent per reaction, the amount of Quant-iT dye used in this protocol is $10 x$ less as we decreased the volume of the reagent to $20 \mu$ l per reaction. Thus, this protocol is very efficient in terms of cost and time used in measurement and preparation. Furthermore, automation of the original manual protocol resulted in higher pipetting precision through avoidance of human error during manual dispensing, increased reproducibility and significant time reduction to perform this assay. Manual pipetting, in particular when using a 384-format plate, is a challenging task where assay accuracy and reproducibility is affected by performance variability between different users preparing the same assay.

The protocol was created to be used with an Eppendorf epMotion 5075. However, it is possible that the same protocol can be adjusted for other automated liquid handling systems.

\section{AUTHOR CONTRIBUTIONS}

Kay Anantanawat performed experiments and drafted the manuscript. Nicola Pitsch performed experiments and edited the manuscript. Caroline Fromont performed experiments and edited the manuscript. Caroline Janitz conceived the concept and experimental design of the study and drafted and revised the manuscript.

\section{ACKNOWLEDGMENTS}

We thank Sylvia Johnson from BMG LABTECH, Australia for her help with the FLUOstar

\section{FINANCIAL \& COMPETING INTERESTS DISCLOSURE}

The NGS facility is funded by Western Sydney University. The authors have no other relevant affiliations or financial involvement with any organization or entity with a financial interest in or financial conflict with the subject matter or materials discussed in the manuscript apart from those disclosed.

No writing assistance was utilized in the production of this manuscript.

\section{OPEN ACCESS}

This work is licensed under the AttributionNonCommercial-NoDerivatives 4.0 Unported License. To view a copy of this license, visit http://creativecommons.org/licenses/ by-nc-nd/4.0/

\section{REFERENCES}

1. Huberman JA. Importance of measuring nucleic acid absorbance at $240 \mathrm{~nm}$ as well as at 260 and $280 \mathrm{~nm}$. Biotechniques 18(4), 636 (1995).

2. Manchester KL. Value of A260/A280 ratios for measurement of purity of nucleic acids. Biotechniques 19(2), 208-210 (1995).

3. Manchester KL. Use of UV methods for measurement of protein and nucleic acid concentrations. Biotechniques 20(6), 968-970 (1996).

4. Le Pecq JB, Paoletti C. A new fluorometric method for RNA and DNA determination. Anal. Biochem. 17(1) 100-107 (1966).

5. Singer VL, Jones LJ, Yue ST, Haugland RP. Characterization of PicoGreen reagent and development of a fluorescence-based solution assay for double-stranded DNA quantitation. Anal. Biochem. 249(2), 228-238 (1997).

6. Quant-IT PicoGreen ds Reagents and kits. www.thermofisher.com/order/catalog/product/P7589

7. Simbolo $M$, Gottardi $M$, Corbo $V$ et al. DNA qualification workflow for next generation sequencing of histopathological samples. PLoS One 8(6), e62692 (2013).

8. Dragan Al, Casas-Finet JR, Bishop ES et al. Characterization of PicoGreen interaction with dsDNA and the origin of its fluorescence enhancement upon binding Biophys J. 99(9), 3010-3019 (2010). 